\title{
Qualidade subjetiva do sono em pacientes com transtorno depressivo
}

\author{
Sarah Laxhmi Chellappa \\ Universität Basel (Suiça) \\ John Fontenele Araujo \\ Universidade Federal do Rio Grande do Norte
}

\begin{abstract}
Resumo
O objetivo deste estudo foi avaliar a qualidade subjetiva do sono em pacientes com transtorno depressivo. Setenta pacientes (44 mulheres e 26 homens) ambulatoriais com diagnóstico de transtorno depressivo foram entrevistados e responderam ao Índice de Qualidade de Sono de Pittsburgh (IQSP), ao Questionário de Hábitos do Sono e ao Inventário de Depressão de Beck (BDI). As médias das pontuações globais do IQSP e BDI foram respectivamente $12,80 \pm 3,77$ e 35,83 $\pm 8,85$, e a correlação foi positiva e significativa entre essas escalas $(r=0,70 ; p<0,05)$. As pontuações globais do IQSP em pacientes com insônia $(16,61 \pm 4,21) \mathrm{e}$ hipersonia $(8,82 \pm 2,71)$ apresentaram diferenças marcantes $(p<0,05)$. Os resultados da análise multivariada indicaram que apenas a insônia foi significativamente associada à qualidade subjetiva do sono $(R C=4,1$; $95 \% I C=2,0-4,2)$. Os resultados sugerem que os pacientes da amostra apresentaram percepção inferior da qualidade subjetiva do sono, principalmente na insônia.
\end{abstract}

Palavras-chave: transtornos do sono; distúrbios do início e da manutenção do sono; distúrbios do sono por sonolência excessiva; transtorno depressivo; saúde mental

\begin{abstract}
Subjective sleep quality in patients with depressive disorder. The aim of this study was to evaluate subjective sleep quality in patients with depressive disorder. Seventy outpatients (44 women and $26 \mathrm{men}$ ) with diagnosis of depressive disorder were interviewed and answered the Pittsburgh Sleep Quality Index (PSQI), the Sleep Habits questionnaire and the Beck Depression Inventory (BDI). Mean PSQI and BDI global scores were $12.80 \pm 3.77$ and $35.83 \pm 8.85$, respectively and correlation was positive and significant between these scales $(r=0.70 ; p<0.05)$. The global PSQI scores of patients with insomnia $(16.61 \pm 4.21)$ and excessive sleepiness $(8.82 \pm 2.71)$ had marked differences $(p<0.05)$. The results of multivariate analysis indicated that only insomnia had a significant association with subjective sleep quality $(O R=4.1 ; 95 \% C I=2.0-4.2)$. These results suggest that patients with depressive disorder of the study sample experienced poor subjective sleep quality, particularly in insomnia.

Keywords: sleep disorders; sleep initiation and maintenance disorders; disorders of excessive somnolence; depressive disorder; menta health
\end{abstract}

$\mathrm{O}$ $\mathrm{s}$ transtornos do sono consistem em uma das principais sintomatologias do transtorno depressivo. Diversos estudos sobre as alterações dos padrões do sono na depressão têm auxiliado sobremaneira na compreensão dos mecanismos neurobiológicos da desregulação do humor no transtorno depressivo (Adrien, 2002; Chellappa \& Araujo, 2006b, 2006c; Riemann \& Voderholzer, 2003). A fim de explicar as anormalidades do sono na depressão, alguns modelos teóricos foram desenvolvidos, como o modelo bi-direcional do sono e da regulação do sono, podendo ser citado, por exemplo, o modelo de deficiência de neurotransmissão. Segundo este, a regulação do sono está associada diretamente à regulação do humor médiante os sistemas de neurotransmissão monoaminérgicos (Riemann \& Voderholzer, 2003).

Um total de $80 \%$ dos pacientes com transtorno depressivo apresenta alterações tanto da quantidade como da qualidade do sono, com queixas freqüentes de insônia, havendo dificuldade de iniciar e manter o sono e/ou despertar precoce pela manhã (Kupfer, 1995). As queixas específicas podem incluir despertares noturnos freqüentes, sono não restaurador, redução do sono total e sonhos perturbadores (Chellappa \& Araújo, 2006b). Em torno de 10 a $20 \%$ dos pacientes com depressão grave ou atípica referem hipersonia como a principal queixa de alteração do sono (Fava, 2004). As queixas de transtornos do sono são correlatos subjetivos do transtorno depressivo e podem representar sintomas pródromos e/ou fatores de risco para o primeiro episódio depressivo (Wirz-Justice \& Van den Hoofdakker, 1999) bem como podem antecipar a recorrência do quadro de transtorno depressivo (Riemann, Berger, \& Voderholzer, 2001). Assim, a avaliação cautelosa dos transtornos do sono nos pacientes com 
transtorno depressivo é importante pela relevância clínica e pelo valor prognóstico (Benca, 2001).

Os transtornos do sono na depressão podem ser avaliados através da percepção subjetiva da qualidade do sono (Agargun, Kara, \& Solmaz, 1997a). Estudos epidemiológicos sugerem a necessidade de acessar a qualidade subjetiva do sono em pacientes deprimidos, (Agargun, Kara, \& Solmaz, 1997b; Mayers, van Hooff, \& Baldwin, 2003), contudo as amostras de estudos precedentes geralmente incluíram pacientes internados, havendo uma escassez de estudos com amostras de pacientes depressivos ambulatoriais.

O objetivo deste estudo foi avaliar a qualidade subjetiva do sono de pacientes ambulatoriais com transtorno depressivo de um hospital geral e examinar a qualidade do sono nos pacientes com insônia e hipersonia.

\section{Método}

\section{Tipo de estudo e participantes}

$\mathrm{O}$ estudo realizado foi do tipo observacional, transversal, incluindo 70 pacientes, sendo $44(62,9 \%)$ mulheres e $26(37,1 \%)$ homens. A média de idade foi 40,48 $\pm 12,54$, e a duração média em meses do transtorno depressivo foi $6,14 \pm 1,90$. Esses participantes eram atendidos no ambulatório de psiquiatria do Hospital Universitário Onofre Lopes da Universidade Federal do Rio Grande do Norte, Natal, RN. Esta instituição é considerada um dos principais hospitais de grande porte no estado do Rio Grande do Norte, para o qual são encaminhados pacientes da capital e de todo o estado.

A seleção dos participantes neste estudo compreendeu os seguintes critérios de inclusão: idade entre 18 e 65 anos e diagnóstico de transtorno depressivo segundo os critérios da American Psychiatric Association (2000), que tem preconizado esse procedimento clínico padrão para definir o diagnóstico do transtorno depressivo. Os critérios diagnósticos do transtorno depressivo incluíram as questões quanto à ocorrência de humor depressivo, à anedonia, às concentração e atenção reduzidas, às alterações no apetite, à irritabilidade, ao sentimento de fracasso, à ideação suicida e aos transtornos do sono. Os cinco psiquiatras responsáveis pelo acompanhamento dos pacientes no referido ambulatório realizaram o diagnóstico. Pacientes com transtorno esquizoafetivo, transtorno bipolar, abuso de substâncias ilícitas e de álcool e transtorno do pânico não foram incluídos nesta pesquisa.

\section{Procedimentos}

As entrevistas foram realizadas pela primeira autora desta pesquisa, devidamente capacitada e treinada na aplicação dos instrumentos. A escolha do hospital foi baseada no fato de ser o principal de nível terciário no Estado do Rio Grande do Norte, para o qual convergem pacientes cujo atendimento requer maior especificidade. Desta maneira, uma grande parcela de pacientes com transtornos do humor, como o transtorno depressivo, é encaminhada ao ambulatório de psiquiatria deste hospital. Assim sendo, a pesquisa foi realizada com indivíduos que apresentavam quadros mais característicos do transtorno depressivo. As entrevistas foram realizadas durante o período de abril a julho de 2005, duas vezes por semana (terças e quintas-feiras), em horário vespertino (13:30-15:30).

A coleta dos dados foi realizada em duas etapas. Primeiramente foi realizada a avaliação médica, e o diagnóstico de transtorno depressivo foi dado através de entrevista clínica por cinco médicos psiquiatras do ambulatório de psiquiatria, responsáveis pelo acompanhamento dos pacientes. Os cinco psiquiatras apresentavam pelo menos dez anos de treinamento em psiquiatria, e estavam capacitados e treinados em detectar e classificar o transtorno depressivo segundo os critérios da American Psychiatric Association (2000).

Para minimizar possíveis vieses de avaliação e garantir a validade interna do estudo, foi realizado um pré-teste com 10 pacientes depressivos para avaliar possíveis dificuldades de compreensão das questões do questionário e para realizar a calibração intra-examinador do pesquisador responsável pelas entrevistas. A calibração foi realizada com o objetivo de adequadamente treinar e capacitar o examinador para a execução da metodologia utilizada. Posteriormente, na etapa de coleta de dados, as escalas de auto-avaliação foram respondidas pelos pacientes com a ajuda de um dos pesquisadores, que explicou de maneira detalhada como responder às escalas utilizadas.

Os questionários utilizados na análise foram aplicados individualmente com apoio do pesquisador e com tempo de duração de 30 a 40 minutos. Os instrumentos foram aplicados de forma coordenada, ou seja, após a leitura e explicação do aplicador sobre o que tratava cada questão e como deveria ser efetuada a resposta. Caso alguma questão não fosse compreendida, o entrevistador repetia a pergunta. Caso o entrevistado não compreendesse a pergunta, a mesma era novamente repetida. Caso não houvesse resposta, o entrevistador passava para a pergunta seguinte.

A pesquisa foi iniciada após a aprovação pelo Comitê de Ética em Pesquisa em Seres Humanos da Universidade Federal do Rio Grande do Norte (№ 111/04). Todos os participantes assinaram o Termo de Consentimento Livre e Esclarecido, antes de participarem da pesquisa, e tiveram sua privacidade respeitada.

\section{Instrumentos}

O Índice da Qualidade do Sono de Pittsburgh (IQSP) foi utilizado para avaliar a qualidade subjetiva do sono (Buysse, Reynolds, \& Monk, 1989) sendo um instrumento com confiabilidade e validade previamente estabelecidas. Este questionário é composto por 19 itens, que são agrupados em sete componentes, cada qual pontuado em uma escala de 0 a 3 . Os componentes são, respectivamente: (1) a qualidade subjetiva do sono; (2) a latência do sono; (3) a duração do sono; (4) a eficiência habitual do sono; (5) as alterações do sono; (6) o uso de medicações para o sono; e (7) a disfunção diurna. Os escores dos sete componentes são somados para conferir uma pontuação global do IQSP, a qual varia de 0 a 21. Pontuações de 0-4 indicam boa qualidade do sono, de 5-10 indicam qualidade ruim e acima de 10 indicam distúrbio do sono (Buysse et al., 1989). Neste estudo, foi utilizada a versão validada deste instrumento em português (Ceolim \& Menna-Barreto, 2000; Xavier, Ferraz, Bertollucci, Poyares, \& Moriguchi, 2001). A confiabilidade do 
IQSP foi indicada pelo Coeficiente Kappa-Ponderado $(K=0,81)$, cujo valor é considerado uma alta concordância intra-examinador (Landis \& Koch, 1977).

O Questionário de Hábitos do Sono é um protocolo validado com 32 itens relacionados aos hábitos do sono dos pacientes, à saúde e ao uso dos estimulantes (Andrade, Benedito-Silva, \& Menna-Barreto, 1992). Dessas 32 questões, 10 são relacionadas à saude do paciente e ao uso de estimulantes e 22 questões são relacionadas ao sono e à presença de alterações do sono. Este questionário foi utilizado para acessar as queixas de transtornos do sono relacionadas à insonia e à hipersonia, sendo usado apenas para classificar os pacientes com insônia, hipersonia ou ausência de um destes transtornos do sono. A insônia foi definida como dificuldade em iniciar o sono, em manter o sono e/ou despertar precoce pela manhã ao menos três vezes por semana, durante os últimos 30 dias, com conseqüências para a vida diária, como, por exemplo, piora da atenção, concentração e desempenho cognitivo. A hipersonia foi definida como o sono prolongado da noite e a sonolência diurna ao menos três vezes por semana nos últimos 30 dias (Sociedade Brasileira do Sono, 2003). A confiabilidade do Questionário de Hábitos do Sono para insônia e hipersonia foi indicada pelo Coeficiente Kappa-Ponderado ( $K$ $=0,76$ ), cujo valor é considerado uma alta concordância intraexaminador (Landis \& Koch, 1977).

O Inventário de Depressão de Beck (Beck Depression Inventory, ou BDI) é um questionário padronizado que permite acessar a severidade do transtorno depressivo, incluindo atitude e sintomas depressivos, além da ideação suicida (Beck, Ward, Mendelson, Mock, \& Erbaugh, 1961). Esta escala consiste de 21 itens, cuja intensidade é indicada por meio de uma pontuação que varia de 0 a 3 . Neste questionário são utilizados os seguintes pontos de corte: menor que $10=$ sem depressão ou depressão mínima; de 10 a 18 = depressão leve a moderada; de 19 a 29 = depressão moderada; de 30 a 63 = depressão grave. Neste estudo foi utilizada versão validada em português (Gorenstein \& Andrade, 1996). A confiabilidade do BDI foi indicada pelo Coeficiente Kappa-Ponderado $(K=0,78)$, cujo valor é considerado uma alta concordância intra-examinador (Landis \& Koch, 1977).

\section{Análise estatística}

Foram realizadas as análises descritivas (freqüências, médias e desvios padrões), o Teste $t$ de Student para amostras independentes para comparação entre médias e a análise de variância (ANOVA), seguida do teste post-hoc de Tukey, para verificar diferença significativa na pontuação do IQSP. Os resultados foram considerados significativos a $p<0,05$. A análise multivariada foi baseada na razão de chances $(R C)$ ajustada, que, por sua vez, foi ajustada pelo método de regressão logística multivariada. Todos os intervalos de confiança $(I C)$ foram incluídos, juntamente com os valores de $R C$. As variáveis como duração do transtorno depressão (com variação de 01 a 12 meses) e a presença de insônia ou hipersonia associada à qualidade do sono (obtida através da pontuação do IQSP) superior ao nível de 0,2 na análise univariada foram incluídas no modelo de regressão logística. Deve ser salientado que a qualidade subjetiva do sono foi obtida através da pontuação do IQSP, sendo categorizada, portanto, de 0-4 como boa qualidade, 5-10 qualidade ruim e acima de 10 como sendo distúrbio do sono. A idade (categorizada em faixas etárias de 10 anos, ou seja, 18-28; 29-39; 40-50, 51 61 e acima de 61 anos), o sexo e o uso de antidepressivos e/ou hipnóticos indutores do sono foram considerados a priori como variáveis de confusão. As análises foram realizadas utilizando o programa EPI-INFO, versão 6,0.

\section{Resultados}

O escore médio geral obtido no Inventário de Depressão de Beck (BDI) foi de 35,83 e o respectivo desvio-padrão foi de $\pm 8,85$. A distribuição dos participantes pela gravidade da depressão segundo os pontos de corte estabelecidos por essa escala é apresentada na Tabela 1.

Tabela 1

Gravidade da depressão, escores do Inventário de Depressão de Beck BDI) respectivas freqüências $(\mathrm{N}=70)$

\begin{tabular}{lccc}
\hline Gravidade da depressão & Pontos de corte do BDI & $n$ & Freqüência (\%) \\
\hline Ausente / mínima & $0-9$ & 0 & 0 \\
Leve & $10-18$ & 4 & 5,7 \\
Moderada & $19-29$ & 26 & 37,1 \\
Grave & $>30$ & 40 & 57,2 \\
\hline
\end{tabular}

A média do Índice de Qualidade do Sono de Pittsburgh (IQSP) obtida na amostra foi $12,80 \pm 3,77$. Uma correlação positiva e significativa foi observada entre o BDI e o IQSP $(r=0,70 ; p<0,05)$. Foram observadas pontuações mais elevadas de quatro componentes deste instrumento: a qualidade subjetiva do sono $(2,47 \pm 0,84)$, a latência do sono $(2,20 \pm 0,68)$, a duração do sono $(2,11 \pm 0,71)$ e a disfunção diurna $(2,56 \pm 0,79)$, respectivamente, componentes $1,2,3$ e 7. A menor pontuação foi referente ao uso de medicação para o sono $(0,81 \pm 0,47)$, seguida pela eficiência habitual do sono $(1,45 \pm 0,61)$ e pelas alterações do sono $(1,68 \pm$ $0,59)$, respectivamente, componentes 6,4 e 5 . Diferenças significativas foram observadas entre os componentes do Índice de Qualidade do Sono de Pittsburgh $(F=31,4 ; p<$ $0,05)$. Nenhuma diferença significativa foi observada entre os componentes 1, 2, 3 e 7. Entretanto, estes componentes tiveram diferenças significativas com relação aos componentes 4,5 e $6(p<0,05)$. 
Neste estudo, 49 (70\%) pacientes apresentavam insônia e $21(30 \%)$ hipersonia. Os pacientes com insônia apresentaram pontuações globais do Índice de Qualidade do Sono significativamente mais elevadas $(16,61 \pm 4,21)$ com relação aos pacientes com hipersonia $(8,82 \pm 2,71)(t=8,97 ; p<0,05)$.

Os pacientes com insônia apresentaram pontuações significativamente mais elevadas dos seguintes componentes: Qualidade subjetiva do sono $(t=3,24 ; p<0,001)$, latência do sono $(t=10,84 ; p<0,001)$, duração do sono $(t=21,33 ; p$ $<0,001)$, eficiência habitual do sono $(t=5,24 ; p<0,001)$ e alterações do sono $(t=13,70 ; p<0,001)$. Estes resultados estão descritos na Tabela 2.
Em relação ao uso de medicações, 27 (38\%) pacientes utilizaram antidepressivos e/ou hipnóticos indutores do sono 15 dias antes das entrevistas, dos quais nove $(13,4 \%)$ utilizaram benzodiazepínicos, três (5\%) amitriptilina, três $(5 \%)$ nortriptilina, três $(5 \%)$ sertralina, dois $(3 \%)$ fluoxetina e quatro $(6,6 \%)$ não recordaram qual a medicação utilizada.

Os resultados da análise multivariada em relação às variáveis idade, sexo, duração da depressão (em meses) e presença de insônia ou hipersonia relacionadas à qualidade subjetiva do sono indicaram que apenas a insônia foi significativamente associada à qualidade do sono $(R C=4,1 ; 95 \% I C=2,0-4,2)$ (ver Tabela 3).

Tabela 2

Comparação de médias (teste t) dos componentes do Índice de Qualidade do Sono de Pittsburgh de pacientes com insônia $(\mathrm{n}=49)$ e pacientes com hipersonia $(\mathrm{n}=21)$

\begin{tabular}{lccc}
\hline \multirow{2}{*}{ Componentes } & \multicolumn{3}{c}{ Pacientes $(N=70)$} \\
\cline { 2 - 4 } & Insônia $^{\text {a }}$ & Hipersonia $^{\text {a }}$ & $p$ \\
\hline Qualidade do sono & $2,6 \pm 0,5$ & $2,1 \pm 0,6$ & 0,004 \\
Latência do sono & $2,9 \pm 0,4$ & $1,0 \pm 0,7$ & $<0,001$ \\
Duração do sono & $2,8 \pm 0,4$ & $1,0 \pm 0,1$ & $<0,001$ \\
Eficiência habitual do sono & $1,8 \pm 0,8$ & $1,1 \pm 0,1$ & $<0,001$ \\
Alterações do sono & $2,4 \pm 0,5$ & $0,9 \pm 0,3$ & $<0,001$ \\
Uso de medicações para o sono & $0,8 \pm 0,9$ & $0,5 \pm 0,7$ & 0,240 \\
Disfunção diurna & $2,8 \pm 0,4$ & $2,4 \pm 0,6$ & 0,140 \\
\hline
\end{tabular}

${ }^{a}$ Média \pm desvio-padrão

Tabela 3

Análises bivariada e multivariada no modelo de regressão logística da qualidade subjetiva do sono e co-variáveis na amostra estudada

\begin{tabular}{lcccccc}
\hline & $R C^{\mathrm{a}}$ & $I C(95 \%)$ & $p$ & $R C^{\mathrm{b}}$ & $I C(95 \%)$ & $p$ \\
\hline Idade $^{\mathrm{c}}$ & 0,7 & $0,4-1,2$ & 0,700 & & \\
Sexo & & & & & \\
$\quad$ Homens & 1,2 & $0,5-1,8$ & 0,800 & & \\
$\quad$ Mulheres & 1,6 & $0,6-1,9$ & 0,080 & & \\
Duração da depressão & ${ }^{\mathrm{d}}$ & 1,4 & $0,7-2,9$ & 0,200 & & \\
Insônia $_{\text {Hipersonia }}$ & 4,5 & $2,0-4,8$ & $<0,001$ & 4,1 & $2,0-4,2$ & $<0,001$ \\
\hline
\end{tabular}

${ }^{\mathrm{a}}$ Razão de chances na análise bivariada

${ }^{\mathrm{b}}$ Razão de chances ajustada no modelo de regressão logística

${ }^{\mathrm{c}}$ Razão de chances relativa à variação do aumento de dez anos na idade (faixa etária)

${ }^{\text {d } R a z a ̃ o ~ d e ~ c h a n c e s ~ r e l a t i v a ~ a ̀ ~ v a r i a c ̧ a ̃ o ~ d o ~ a u m e n t o ~ d e ~ u m ~ m e ̂ s ~ n a ~ d u r a c ̧ a ̃ o ~ d a ~ d e p r e s s a ̃ o ~}$ 


\section{Discussão}

Neste estudo, foram observadas pontuações mais elevadas da qualidade subjetiva do sono, da latência do sono, da eficiência do sono e da disfunção diurna, indicando pior qualidade do sono na amostra (DiMatteo, Lepper, \& Croghan, 2003) em comparação com indivíduos sadios, cujas pontuações são inferiores a 5, indicativas de boa qualidade do sono. As pontuações elevadas do Inventário de Depressão de Beck indicaram que a depressão grave foi freqüente entre os pacientes. Simultaneamente, a correlação significativa entre o índice da qualidade do sono de Pittsburgh e o Inventário de Depressão de Beck indica que uma pior qualidade subjetiva do sono foi associada à gravidade da depressão nos pacientes. Os pacientes depressivos com insônia apresentaram um pior perfil da qualidade do sono em relação aos pacientes com hipersonia, principalmente nos componentes relacionados à qualidade do sono, à latência do sono, à duração do sono, à eficiência habitual do sono, às alterações do sono.

A importância de se realizar um estudo quanto à qualidade subjetiva do sono reside na sua relevância clínica. Ao se determinar a percepção do sono, permite-se delinear, primeiramente, a existência de depressão e, posteriormente, a propria gravidade do quadro depressivo. Estudos com populações clínicas geralmente indicam uma elevada co-morbidade entre a insônia e os transtornos psiquiátricos, notadamente em episódios de depressão (Ford \& Cooper-Patrick, 2001; Tylee, Gastpar, Lepine, \& Mendlewicz, 1999). Paralelamente, tem sido descrito que pacientes com insônia relatam freqüentemente a eficiência diminuída do sono, bem como alterações do sono e uma pior qualidade do sono (Agargun et al., 1997a). Desta forma, a insônia pode estar estreitamente associada à patogênese da depressão (Adrien, 2002).

Os estudos do sono sugerem que a desregulação do sono pode ocorrer nos primeiros estágios da depressão, antecipá-la ou ser um sintoma residual da depressão não responsiva ao manejo terapêutico utilizado (Chellappa \& Araújo, 2006c). Diversas hipóteses podem explicar os mecanismos subjacentes do sono, da qualidade do sono, e do transtorno depressivo. O sistema serotoninérgico é envolvido na regulação do ciclo sono-vigília, estando sua atividade no ápice durante o estado de vigília e no mínimo durante o sono. Assim sendo, a serotonina é um neurotransmissor com um papel fundamental na regulação do sono (Linnoila \& Virkkunen, 1992). Durante o episódio depressivo, ocorre uma diminuição funcional da serotonina, que repercute em alterações específicas do sono, notadamente na insônia (Sateia \& Nowell, 2004). Paralelamente, manipulações do ciclo sono-vigília podem induzir sintomas depressivos (Cano-Lozano, Espinosa-Fernandez, Miro \& Buela-Casal, 2003; Riemann et al., 2001). Desta forma, a forte relação bidirecional entre sono, transtornos do sono e transtorno depressivo desempenha um papel fundamental que pode explicar os resultados obtidos neste estudo e enfatiza a importância de avaliar queixas do sono e a qualidade subjetiva do sono em pacientes depressivos (Chellappa \& Araújo, 2006a).

Em relação às limitações do estudo, dificuldades foram encontradas quanto ao número de respondentes $(N=70)$, o que restringe a generalização dos dados. Futuros estudos com um número maior de participantes são sugeridos para melhor avaliar a recorrência e o tempo de surgimento dos distúrbios do sono.

Os resultados sugerem que os pacientes com transtorno depressivo apresentaram um pior perfil da qualidade subjetiva do sono, principalmente aqueles do grupo com insônia. Desta forma, os transtornos e a percepção da qualidade do sono parecem desempenhar uma função importante na depressão e devem conseqüentemente ser adequadamente examinados durante a avaliação clínica.

\section{Referências}

Adrien, J. (2002). Neurobiological bases for the relation between sleep and depression. Sleep Medicine Reviews, 6(5), 341-351.

Agargun, M. Y., Kara, H., \& Solmaz, M. (1997a). Sleep disturbance and suicidal behavior in patients with major depression. Journal of Clinical Psychiatry, $58(6), 249-251$.

Agargun, M. Y., Kara, H., \& Solmaz, M. (1997b). Subjective sleep quality and suicidality in patients with major depression. Journal of Psychiatry Research, 31(3), 377-381.

American Psychiatric Association. (2000). Diagnostic and statistical manual

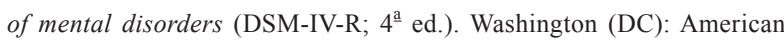
Psychiatric Press.

Andrade, M. M., Benedito-Silva, A. A., \& Menna-Barreto, L. (1992). Correlations between morningness-eveningness character, sleep habits and temperature rhythm. Brazilian Journal of Medical and Biological Research, 25(8), 835-839.

Beck, A. T., Ward, C. H., Mendelson, M., Mock, J., \& Erbaugh, J. (1961). An inventory for measuring depression. Archives of General Psychiatry, 4, 561-571.

Benca, R. M. (2001) Consequences of insomnia and its therapies. Journal of Clinical Psychiatry, 62(10), 33-38.

Buysse, D. J, Reynolds, C. F. 3rd, Monk, T. H., Berman, S. R., \& Kupfer, D. J. (1989). The Pittsburgh Sleep Quality Index: a new instrument for psychiatric practice and research. Psychiatry Research, 28(2), 193-213.

Cano-Lozano, M. C., Espinosa-Fernandez, L., Miro, E., \& Buela-Casal, G. (2003). Una revisión de las alteraciones del sueño en la depresión. Revista de Neurología, 36(4), 366-375.

Ceolim, M. F., \& Menna-Barreto, L. (2000). Sleep/wake cycle and physica activity in healthy elderly people. Sleep Research Online, 3, 87-95.

Chellappa, S. L., \& Araújo, J. F. (2006a). Excessive daytime sleepiness in patients with depressive disorder. Revista Brasileira de Psiquiatria, 28(2), 126-130.

Chellappa, S. L., \& Araújo, J. F. (2006b). Relevância clínica de pesadelos em pacientes com transtorno depressivo. Revista de Psiquiatria Clínica, 33(4), 183-187.

Chellappa, S. L., \& Araújo, J. F. (2006c). Transtornos do sono em pacientes ambulatoriais com depressão. Revista de Psiquiatria Clínica, 33(5), 233238.

DiMatteo, M. R., Lepper, H. S., \& Croghan, T. W. (2000). Depression is a risk for noncompliance with medical treatment: meta-analysis of the effects of anxiety and depression on patient adherence. Archives of Internal Medicine, 160(14), 2101-2107.

Fava, M. (2004). Daytime sleepiness and insomnia as correlates of depression. Journal of Clinical Psychiatry, 65(16), 27-32.

Ford, D. E., Cooper-Patrick, L. (2001). Sleep disturbances and mood disorders: an epidemiologic perspective. Depression and Anxiety, 14(1), 3-6.

Gorenstein, C., \& Andrade, L. (1996). Validation of a Portuguese version of the Beck Depression Inventory and the State-Trait Anxiety Inventory in Brazilian subjects. Brazilian Journal of Medical and Biological Research, 
29(4), 453-437.

Kupfer, D. J. (1995) Sleep research in depressive illness: clinical implications - a tasting menu. Biological Psychiatry, 38(6), 391-403.

Landis, J. R., \& Koch, G. G. (1977). The measurement of observer agreement for categorical data. Biometrics, 33(1), 159-174.

Linnoila, V. M., \& Virkkunen, M. (1992). Aggression, suicidality, and serotonin. Journal of Clinical Psychiatry, 53, 46-51.

Mayers, A. G., van Hooff, J. C., \& Baldwin, D. S. (2003). Quantifying subjective assessment of sleep and life-quality in antidepressant-treated depressed patients. Human Psychopharmacology, 18(1), 21-27.

Riemann, D., Berger, M., \& Voderholzer, U. (2001). Sleep and depression results from psychobiological studies: an overview. Biological Psychiatry, 57(1), 67-103.

Riemann, D., \& Voderholzer, U. (2003). Primary insomnia: a risk factor to develop depression? Journal of Affective Disorders, 76(1), 255-259.
Sociedade Brasileira de Sono. (2003). I Consenso Brasileiro sobre Insônia. Hypnós, 4, 3-45.

Sateia, M., \& Nowell, P. D. (2004). Insomnia. Lancet, 364, 1959-1973.

Tylee, A., Gastpar, M., Lepine, J. P., \& Mendlewicz, J. (1999). DEPRES II (Depression Research in European Society II): a patient survey of the symptoms, disability and current management of depression in the community. DEPRES Steering Committee. Journal of Clinical Psychopharmacology, 14(3), 139-151.

Xavier, F., Ferraz, M., Bertollucci, P., Poyares, D., \& Moriguchi, E. H. (2001). Episódio depressivo maior, prevalência e impacto sobre a qualidade de vida, sono e cognição em octogenários. Revista Brasileira de Psiquiatria, $23(2), 62-70$

Wirz-Justice, A., \& Van den Hoofdakker, R. H. (1999) Sleep deprivation in depression: what do we know, where do we go? Biological Psychiatry, 46(4), 445-453.

Sarah Laxhmi Chellappa, mestre em Ciências da Saúde pela Universidade Federal do Rio Grande do Norte, é doutoranda em Neurociências na Universität Basel (Suiça). E-mail: sarah.chellappa@upkbs.ch

John Fontenele Araujo, doutor em Neurociência e Comportamento pela Universidade de São Paulo, é professor associado no Departamento de Fisiologia, Centro de Biociências, Universidade Federal do Rio Grande do Norte. Endereço para correspondência: Caixa Postal 1506; Natal, RN; CEP: 59078-970. Tel./ Fax: (84) 3615-1264. E-mail: araujo@cb.ufrn.br 